\title{
ON NONUNIQUENESS FOR THE ANISOTROPIC CALDERÓN PROBLEM WITH PARTIAL DATA
}

\author{
THIERRY DAUDÉ ${ }^{1}$, NIKY KAMRAN ${ }^{2}$ and FRANÇOIS NICOLEAU ${ }^{3}$ \\ ${ }^{1}$ Département de Mathématiques, UMR CNRS 8088, Université de Cergy-Pontoise, \\ 95302 Cergy-Pontoise, France; \\ email: thierry.daude@u-cergy.fr \\ ${ }^{2}$ Department of Mathematics and Statistics, McGill University, Montreal, QC, H3A 0B9, Canada; \\ email: nkamran@math.mcgill.ca \\ ${ }^{3}$ Laboratoire de Mathématiques Jean Leray, UMR CNRS 6629, 2 Rue de la Houssinière BP \\ 92208, F-44322, Nantes Cedex 03, France; \\ email: francois.nicoleau@univ-nantes.fr
}

Received 8 May 2019; accepted 10 December 2019

\begin{abstract}
We show that there is nonuniqueness for the Calderón problem with partial data for Riemannian metrics with Hölder continuous coefficients in dimension greater than or equal to three. We provide simple counterexamples in the case of cylindrical Riemannian manifolds with boundary having two ends. The coefficients of these metrics are smooth in the interior of the manifold and are only Hölder continuous of order $\rho<1$ at the end where the measurements are made. More precisely, we construct a toroidal ring $(M, g)$ and we show that there exist in the conformal class of $g$ an infinite number of Riemannian metrics $\tilde{g}=c^{4} g$ such that their corresponding partial Dirichlet-to-Neumann maps at one end coincide. The corresponding smooth conformal factors are harmonic with respect to the metric $g$ and do not satisfy the unique continuation principle.
\end{abstract}

2010 Mathematics Subject Classification: 81U40, 35P25 (primary); 58 J50 (secondary)

\section{Introduction}

1.1. The anisotropic Calderón problem with partial data. The anisotropic Calderón problem on compact connected Riemannian manifolds with boundary is one of the most famous examples of inverse problems for an elliptic equation. The original problem that Calderón considered was whether one can recover the physical properties of a medium (like its electrical conductivity) by making only

(c) The Author(s) 2020. This is an Open Access article, distributed under the terms of the Creative Commons Attribution licence (http://creativecommons.org/licenses/by/4.0/), which permits unrestricted re-use, distribution, and reproduction in any medium, provided the original work is properly cited. 
voltage and current measurements at its boundary. This latter problem can be naturally formulated as a problem of geometric analysis in terms of the Dirichletto-Neumann map (DN map in short), for the Laplace-Beltrami operator on Riemannian manifolds with boundary. We refer to surveys [18, 30, 41, 44] for a description of the current state of the art on the general anisotropic Calderón problem and also to $[2,4,10,11,16,17,24,25,27-29,31,33,34,36]$ for important contributions to the question of uniqueness. On one hand, the uniqueness issue in the Calderón problem is still far from being completely understood in the case of smooth Riemannian manifolds of dimension greater than or equal to three and remains a major open problem. On the other hand, some counterexamples to uniqueness in the case in which the Dirichlet and Neumann data are measured on disjoint subsets of the boundary were found in our recent papers [6-8].

The main goal of this paper is to give some nonuniqueness results for the anisotropic Calderón problem with partial data (that is, in the case where the Dirichlet and Neumann measurements are made on the same open subset $\Gamma$ of the boundary), for a class of metrics whose coefficients are smooth in the interior of the manifold and Hölder continuous on the subset of the boundary where the measurements are made.

In order to state our main result, let us first recall the definition of the DN map (see for instance [36] for the geometric formulation of the DN map for smooth Riemannian manifolds that we use here and [1] for the formulation of the DN map corresponding to the original Calderón problem in terms of anisotropic conductivities with coefficients, which are only $L^{\infty}$ ). Let $M$ be an $n$-dimensional smooth compact connected manifold with smooth boundary $\partial M$. We assume that this manifold $M$ is equipped with a Riemannian metric $g=\left(g_{i j}\right)$ with measurable bounded coefficients satisfying (in local coordinates) the uniform ellipticity condition

$$
\sum_{i, j} g^{i j}(x) \xi_{i} \xi_{j} \geqslant c|\xi|^{2} \quad \text { for a.e } x \in M \text { and } \xi \in \mathbb{R}^{n}
$$

where the constant $c$ is strictly positive and where $\left(g^{i j}\right)$ is the inverse of $\left(g_{i j}\right)$.

On the Riemannian manifold $(M, g)$, we consider the Laplace-Beltrami operator $\Delta_{L B}$ given in local coordinates by

$$
\Delta_{L B}=-\Delta_{g}=-\frac{1}{\sqrt{|g|}} \partial_{i}\left(\sqrt{|g|} g^{i j} \partial_{j}\right)
$$

where $|g|=\operatorname{det}\left(g_{i j}\right)$ is the determinant of the metric tensor $\left(g_{i j}\right)$ and where we use the Einstein summation convention. It is well known that the Laplace-Beltrami 
operator $-\Delta_{g}$ with Dirichlet boundary conditions on $\partial M$ is self-adjoint on $L^{2}(M$, $d \mathrm{Vol}_{g}$ ) and that 0 is not an eigenvalue of $-\Delta_{g}$.

Let us consider the Dirichlet problem

$$
\begin{cases}-\Delta_{g} u=0, & \text { on } M, \\ u=\psi, & \text { on } \partial M .\end{cases}
$$

A classical result (see for instance $[1,13,41,43]$ ) ensures that for any $\psi \in$ $H^{1 / 2}(\partial M)$, there exists a unique weak solution $u \in H^{1}(M)$ of the Dirichlet problem (1.2). We recall that $u$ is a weak solution of (1.2) if

$$
\int_{M}\langle d u, d w\rangle_{g} d \operatorname{Vol}_{g}=0 \quad \text { for all } w \in H_{0}^{1}(M),
$$

where $\langle d u, d w\rangle_{g}$ is the pointwise scalar product of the 1 -forms $d u, d v$ on $M$ induced by $g$ and given in local coordinates by $\langle d u, d w\rangle_{g}=g^{i j} \partial_{i} u \partial_{j} w$, and if the trace of $u$ on the boundary is equal to $\psi$. So, we can define the DN map as an operator $\Lambda_{g}$ from $H^{1 / 2}(\partial M)$ to $H^{-1 / 2}(\partial M)$ by

$$
\left\langle\Lambda_{g} \psi \mid \phi\right\rangle=\int_{M}\langle d u, d v\rangle_{g} d \operatorname{Vol}_{g}, \quad \text { for all } \psi, \phi \in H^{1 / 2}(\partial M),
$$

where $u$ is the unique weak solution of the Dirichlet problem (1.2), $v$ is any element of $H^{1}(M)$ such that $v_{\mid \partial M}=\phi$, and $\langle\cdot \mid \cdot\rangle$ is the standard $L^{2}$ duality pairing between $H^{1 / 2}(\partial M)$ and its dual. In the case where the metric $g$ and the function $\psi$ are smooth, this definition coincides with the usual one

$$
\Lambda_{g}(\psi)=\left(\partial_{\nu} u\right)_{\mid \partial M},
$$

where $\left(\partial_{\nu} u\right)_{\mid \partial M}$ is the normal derivative of $u$ with respect to the unit outer normal $v$ on $\partial M$.

As mentioned earlier, we are interested in the case in which the Dirichlet and Neumann data are measured on the same nonempty open subset $\Gamma$ of the boundary $\partial M$. Let us introduce the subspace of $H^{1 / 2}(\partial M)$ defined by

$$
H_{c o}^{1 / 2}(\Gamma)=\left\{f \in H^{1 / 2}(\partial M) \mid \operatorname{supp} f \subset \Gamma\right\} .
$$

The partial DN map is defined in a weak formulation as the operator $\Lambda_{g, \Gamma}$ such that

$$
\left\langle\Lambda_{g, \Gamma}(\psi) \mid \phi\right\rangle=\int_{M}\langle d u, d v\rangle_{g} d \mathrm{Vol}_{g}, \quad \text { for all } \psi, \phi \in H_{c o}^{1 / 2}(\Gamma),
$$

where $u$ is the unique weak solution of the Dirichlet problem (1.2) and where $v$ is any element of $H^{1}(M)$ such that $v_{\mid \partial M}=\phi$. As previously, for smooth metrics $g$ and smooth boundary data $\psi$, the partial DN map is simply given by

$$
\Lambda_{g, \Gamma}(\psi)=\left(\partial_{\nu} u\right)_{\mid \Gamma} .
$$


In its simplest form, the anisotropic Calderón problem with partial data can be stated as follows:

If a pair of partial DN maps $\Lambda_{g_{1}, \Gamma}$ and $\Lambda_{g_{2}, \Gamma}$ coincide, is it true that the metrics $g_{1}$ and $g_{2}$ are the same?

Because of several natural and geometric gauge invariances, the answer to the question stated above turns out to be negative. These lead to refined formulations of the Calderón problem that we shall present shortly and that constitute the actual statement of this inverse problem. Indeed, it results from definition (1.2)-(1.7) that the partial DN map $\Lambda_{g, \Gamma}$ is invariant when the metric $g$ is pulled back by any diffeomorphism of $M$ whose restriction to $\Gamma$ is the identity, that is,

$$
\forall \phi \in \operatorname{Diff}(M) \quad \text { such that } \phi_{\mid \Gamma}=\mathrm{Id}, \quad \Lambda_{\phi^{*} g, \Gamma}=\Lambda_{g, \Gamma} .
$$

In dimension two, there is another gauge invariance of the DN map due to the conformal invariance of the Laplace-Beltrami operator. More precisely, recall that in dimension two,

$$
\Delta_{c g}=\frac{1}{c} \Delta_{g}
$$

for any smooth function $c>0$. Therefore, we have in dimension two

$$
\forall c \in C^{\infty}(M), \quad c>0 \quad \text { and } \quad c_{\mid \Gamma}=1, \quad \Lambda_{c g, \Gamma}=\Lambda_{g, \Gamma}
$$

since the unit outer normal vectors $v_{c g}$ and $v_{g}$ are identical on $\Gamma$.

It follows that the appropriate question (called the anisotropic Calderón conjecture with partial data) to address is the following:

Let $M$ be an n-dimensional smooth compact connected manifold with smooth boundary $\partial$ M. Let $g, \tilde{g}$ denote Riemannian metrics on $M$ with measurable bounded coefficients and let $\Gamma$ be an open subset of $\partial M$. Suppose that

$$
\Lambda_{g, \Gamma}=\Lambda_{\tilde{g}, \Gamma} .
$$

Does it follow that $g=\tilde{g}$ up to gauge invariance (1.9) if $n \geqslant 3$ and up to gauge invariances (1.9) and (1.10) in dimension $n=2$ ?

One may also consider a simpler inverse problem by assuming that the Riemannian manifolds $(M, g)$ and $(M, \tilde{g})$ belong to the same conformal class, that is, $\tilde{g}=c g$ for some positive smooth function $c$ on $M$. In that case, the anisotropic Calderón problem amounts to the following statement:

Let $M$ be an $n$-dimensional smooth compact connected manifold with smooth boundary $\partial M$. Let $g$ denote a Riemannian metric on $M$ with measurable bounded coefficients and let $\Gamma$ be an open subset of $\partial M$. Let c be a smooth positive function on M. If

$$
\Lambda_{c g, \Gamma}=\Lambda_{g, \Gamma}
$$


then is it true that

$$
c=1, \quad \text { on } M ?
$$

In fact, according to (1.9), the assumption $\Lambda_{c g, \Gamma}=\Lambda_{g, \Gamma}$ should entail the question: Does there exist a diffeomorphism $\phi: M \longrightarrow M$ with $\phi_{\mid \Gamma}=$ Id such that

$$
\phi^{*} g=c g ?
$$

But, as was proved by Lionheart [37] for smooth metrics, any diffeomorphism $\phi: M \longrightarrow M$ that satisfies $\phi^{*} g=c g$ and $\phi_{\mid \Gamma}=$ Id for a nonempty open subset $\Gamma$ of $\partial M$ is the identity on the whole manifold $M$. Thus, condition (1.12) may therefore be replaced by condition (1.11).

Finally, there exists the last version of the anisotropic Calderón problem with partial data on $\Gamma$ involving an external potential. Consider the Dirichlet problem for the Schrödinger equation on $(M, g)$ with potential $V \in L^{\infty}(M)$

$$
\begin{cases}\left(-\Delta_{g}+V\right) u=0, & \text { on } M, \\ u=\psi, & \text { on } \partial M,\end{cases}
$$

where $\psi \in H_{c o}^{1 / 2}(\Gamma)$. We assume that 0 does not belong to the Dirichlet spectrum of $-\Delta_{g}+V$. Then, there exists a unique weak solution $u \in H^{1}(M)$ of (1.13) (see for instance $[\mathbf{1 0}, 41])$. As previously, this allows us to define in the same way (that is, in a weak sense) the partial DN map $\Lambda_{g, V, \Gamma}(\psi)$ for all $\psi \in H_{c o}^{1 / 2}(\Gamma)$.

For smooth Riemannian metrics $g$, it is well known that there is a close connection between the anisotropic Calderón problem for Schrödinger operators and the anisotropic Calderón problem within the conformal class of a fixed metric $g$. It is based on the transformation law for the Laplace-Beltrami operator on an $n$ dimensional Riemannian manifold $(M, g)$ under conformal changes of the metric, that is,

$$
-\Delta_{c^{4} g} u=c^{-(n+2)}\left(-\Delta_{g}+q_{g, c}\right)\left(c^{n-2} u\right),
$$

where the potential $q_{g, c}$ is given by

$$
q_{g, c}=c^{-n+2} \Delta_{g} c^{n-2}
$$

As a by-product, we get for instance the following result for smooth metrics (see [7], Proposition 1.1).

Proposition 1.1. Let $\Gamma$ be any fixed open set of $\partial$. Assume that $c$ is a smooth positive function on $M$ such that $c=1$ on $\Gamma$ and $\partial_{\nu} c^{n-2}=0$ on $\Gamma$. Then

$$
\Lambda_{c^{4} g, \Gamma}=\Lambda_{g, q_{g, c}, \Gamma}
$$


In particular, if the potential $q_{g, c}=0$, that is, if the conformal factor $c$ satisfies additionally

$$
\Delta_{g} c^{n-2}=0, \quad \text { on } M,
$$

we get immediately

$$
\Lambda_{c^{4} g, \Gamma}=\Lambda_{g, \Gamma}
$$

Note that in dimension $n=2,(1.17)$ is automatically satisfied and (1.16) corresponds simply to gauge invariance (1.12). In dimension $n \geqslant 3$ and for smooth metrics $g$, the unique continuation principle implies that $c=1$ on $M$ (recall that not only (1.17) is satisfied, but $c$ must be identically 1 on $\Gamma$ and $\partial_{\nu} c$ must be 0 on $\Gamma$ ). Thus, if we want to use the result in Proposition 1.1 to obtain a counterexample to uniqueness for the anisotropic Calderón problem with partial data, we need to construct a metric $g$ in such a way that the Laplace-Beltrami operator $-\Delta_{g}$ does not satisfy the unique continuation principle.

We recall that, in dimension $n \geqslant 3$, the unique continuation principle holds for a uniformly elliptic operator on a domain $\Omega$ if the coefficients of the principal part of this operator are locally Lipschitz continuous, whereas in dimension $n=2$, the unique continuation principle holds if the coefficients of the principal part are $L^{\infty}$ (see for instance [21, 22, 42]). Nevertheless, in dimension $n=3$, if the coefficients of the principal part are only Hölder continuous, there exist examples of nonunique continuation. The first such example was given in 1963 by Pliś [40], and later in 1972, a sharper counterexample was found by Miller [39] for an elliptic equation in divergence form. This divergence form is very well adapted to our Riemannian setting. So, the main and basic idea of our paper is to construct a metric $g$ on a suitable manifold $M$ such that the Laplacian $\Delta_{g}$ is nothing but Miller's elliptic operator and the conformal factor $c$ is very close to Miller's solution.

But, before giving this construction, our first task is to slightly extend Proposition 1.1 for metrics $g$ having coefficients in $L^{\infty}(M)$ since, in this case, the potential $q_{g, c}$ only has a distributional sense. In other words, we have to write Proposition 1.1 (with $q_{g, c}=0$ ) in a weak sense. To do this, we remark that, for a smooth metric $g$ and for a smooth conformal factor $c$, the conditions $\partial_{\nu} c=0$ on $\Gamma$ and $\Delta_{g} c^{n-2}=0$ on $M$ are equivalent to

$$
\int_{M}\left\langle d\left(c^{n-2}\right), d w\right\rangle_{g} d \operatorname{Vol}_{g}=0, \quad \forall w \in H^{1}(M) \text { such that supp } w_{\mid \partial M} \subset \Gamma,
$$

thanks to Green's formula

$$
\int_{M} \Delta_{g}\left(c^{n-2}\right) w d \operatorname{Vol}_{g}+\int_{M}\left\langle d\left(c^{n-2}\right), d w\right\rangle_{g} d \operatorname{Vol}_{g}=\int_{\partial M} \partial_{\nu} c^{n-2} w d \sigma_{g},
$$


where $d \mathrm{Vol}_{g}$ denotes the Riemannian volume element and $d \sigma_{g}$ denotes the volume element induced by $g$ on $\partial M$.

Now, we can state the following extension of Proposition 1.1 for metrics with bounded measurable coefficients, which is one of the main arguments of our counterexamples for the anisotropic Calderón problem with partial data.

Proposition 1.2. Let $\Gamma$ be any fixed open set of $\partial M$. Assume that $c$ is a smooth positive function on $M$ such that $c=1$ on $\Gamma$ and such that

$$
\int_{M}\left\langle d\left(c^{n-2}\right), d w\right\rangle_{g} d \operatorname{Vol}_{g}=0, \quad \forall w \in H^{1}(M) \text { such that supp } w_{\mid \partial M} \subset \Gamma .
$$

Then,

$$
\Lambda_{c^{4} g, \Gamma}=\Lambda_{g, \Gamma}
$$

Proof. For any $\psi, \phi \in C_{0}^{\infty}(\Gamma)$, the partial DN map $\Lambda_{c^{4} g, \Gamma}$ is given by the relation

$$
\left\langle\Lambda_{c^{4} g, \Gamma} \psi \mid \phi\right\rangle=\int_{M}\langle d u, d w\rangle_{c^{4} g} d \mathrm{Vol}_{c^{4} g},
$$

where $u \in H^{1}(M)$ is the unique weak solution of (1.2) associated with the metric $c^{4} g$ with $u_{\mid \partial M}=\psi$ and $w \in C^{\infty}(M)$ is any extension of $\phi$. Note that the existence of such an extension is given for instance in monograph [35], Corollary 6.27, together with the Tietze extension theorem. As a consequence, the function $c^{n-2} u w \in H^{1}(M)$ and its trace on the boundary has its support in $\Gamma$. Now, a straightforward algebraic calculation gives

$$
\begin{aligned}
\int_{M}\langle d u, d w\rangle_{c^{4} g} d \operatorname{Vol}_{c^{4} g}= & \int_{M}\left\langle d\left(c^{n-2} u\right), d\left(c^{n-2} w\right)\right\rangle_{g} d \operatorname{Vol}_{g} \\
& -\int_{M}\left\langle d\left(c^{n-2}\right), d\left(c^{n-2} u w\right)\right\rangle_{g} d \operatorname{Vol}_{g} .
\end{aligned}
$$

Thus, thanks to hypothesis (1.20), we get

$$
\left\langle\Lambda_{c^{4} g, \Gamma} \psi \mid \phi\right\rangle=\int_{M}\left\langle d\left(c^{n-2} u\right), d\left(c^{n-2} w\right)\right\rangle_{g} d \operatorname{Vol}_{g} .
$$

Let us now prove that $v=c^{n-2} u$ is a weak solution of (1.2). Indeed, since $u$ is the unique weak solution of (1.2) associated with the metric $c^{4} g$, we have for any $\varphi \in C_{0}^{\infty}(M)$

$$
\int_{M}\langle d u, d \varphi\rangle_{c^{4} g} d \operatorname{Vol}_{c^{4} g}=0
$$


Using again relation (1.23) (with $w$ replaced by $\varphi$ ) and (1.18), we get for any $\varphi \in C_{0}^{\infty}(M)$,

$$
\int_{M}\left\langle d\left(c^{n-2} u\right), d\left(c^{n-2} \varphi\right)\right\rangle_{g} d \operatorname{Vol}_{g}=0 .
$$

It follows that $v=c^{n-2} u$ is a weak solution of (1.2) for the metric $g$, which satisfies $v_{\mid \partial M}=\psi$ since $c=1$ on $\Gamma$.

Then, using the definition of the partial DN map $\Lambda_{g, \Gamma}$ and (1.24) again, we get immediately

$$
\left\langle\Lambda_{c^{4} g, \Gamma} \psi \mid \phi\right\rangle=\left\langle\Lambda_{g, \Gamma} \psi \mid \phi\right\rangle \quad \text { for all } \psi, \phi \in C_{0}^{\infty}(\Gamma) .
$$

We conclude the proof by a standard density argument.

1.2. Statement of the main result. Let us introduce first some notations. We consider the $n$-dimensional manifold

$$
M=[0,1] \times T^{n-1},
$$

where $T^{n-1}$ denotes the $(n-1)$-dimensional torus $(n \geqslant 3)$. This manifold has the topology of a cylinder. Using the standard toroidal coordinates, we can also interpret $M$ as a toroidal ring (see [12], Remark 2.5). Note that the boundary of $M$ is disconnected and consists in the disjoint union of two copies of $T^{n-1}$ (which we call ends in this paper), more precisely,

$$
\partial M=\Gamma_{0} \cup \Gamma_{1}, \quad \Gamma_{0}=\{0\} \times T^{n-1}, \quad \Gamma_{1}=\{1\} \times T^{n-1} .
$$

Our main result is the following.

THEOREM 1. There exists a Riemannian metric $g$ on $M$ whose coefficients are smooth in $[0,1) \times T^{n-1}$ and Hölder continuous of order $\rho<1$ on the end $\Gamma_{1}$, and there exist an infinite number of smooth positive conformal factors $c$ that are not identical to 1 on $M$ such that the following partial DN maps on $\Gamma_{1}$ are identical:

$$
\Lambda_{c^{4} g, \Gamma_{1}}=\Lambda_{g, \Gamma_{1}} .
$$

As we have mentioned earlier, the proof of this theorem is rather simple and relies on Miller's famous counterexample to unique continuation for a uniformly elliptic equation in divergence form in dimension three (see [39]). Assume for a moment that the dimension of our manifold $M$ is three, and let us summarize the strategy of the proof. We consider first a metric $g$ on $M$ such that $\Delta_{g}$ is precisely the uniformly elliptic operator from Miller's construction. Note in passing that the 
elliptic operator from [39] naturally lives on the cylinder $M=[0,1] \times T^{2}$ as was observed by Gianotti in [12]. Then, using Miller's solution of this elliptic partial differential equation (PDE), we shall construct in Section 2 an infinite family of smooth conformal factors $c$ satisfying the assumptions of Proposition 1.2, that is, $c=1$ on $\Gamma_{1}$ and $\Delta_{g} c^{n-2}=0, \partial_{\nu} c=0$ on $\Gamma_{1}$ in the following weak sense:

$$
\int_{M}\left\langle d\left(c^{n-2}\right), d w\right\rangle_{g} d \operatorname{Vol}_{g}=0, \quad \forall w \in H^{1}(M) \text { such that supp } w_{\mid \partial M} \subset \Gamma_{1} .
$$

This leads automatically to counterexamples to uniqueness for the Calderón problem with partial data in dimension three since the metrics $g$ and $c^{4} g$ are not isometric (see the proof of Theorem 1). The proof in the case of higher dimensions is similar.

REMARK 1.1. It is important to stress that, even though two of the coefficients (namely, $A_{1}(t)$ and $A_{3}(t)$ ) of the elliptic operator $\Delta_{g}$ are only Hölder continuous functions, $\Delta_{g} c^{n-2}$ is classically well defined since these two functions are not differentiated with respect to $t$ in the expression of $\Delta_{g}$ (see [39], Theorem 1, or Proposition 2 in this paper). In other words, the equation $\Delta_{g} c^{n-2}=0$ on $M$ can be also understood in the classical sense.

REMARK 1.2. All the derivatives of the conformal factors $c$ at the end $\Gamma_{1}$ are equal to zero as one would expect from boundary determination results (see [26]).

REMARK 1.3. We emphasize that this theorem is of a local nature. We cannot obtain with the same approach a counterexample for the anisotropic Calderón problem with global data, that is, when $\Gamma=\partial M$. Indeed, if the smooth conformal factor satisfies $c=1$ on $\partial M$ and

$$
\int_{M}\left\langle d\left(c^{n-2}\right), d w\right\rangle_{g} d \operatorname{Vol}_{g}=0, \quad \forall w \in H^{1}(M),
$$

then, choosing $w=c^{n-2}$ in (1.30), we obtain immediately that $c$ is identical to 1 on $M$. An alternative interpretation is to say that 0 is not a Dirichlet eigenvalue of the Laplace-Beltrami operator $\Delta_{g}$.

\subsection{A brief history of known results on the anisotropic Calderón problem.}

In this last part of the Introduction, we give a brief and nonexhaustive survey of some of the most important known results on the anisotropic Calderón conjecture.

In dimension two, the anisotropic Calderón conjecture for global and partial data has been settled positively for compact connected Riemannian surfaces 
in $[34,36]$. We also refer to $[2,4]$ for similar results for global and partial data in the case of anisotropic conductivities, which are only $L^{\infty}$ on bounded domains of $\mathbb{R}^{2}$. In dimension $n \geqslant 3$, if the Riemannian manifold is real analytic, compact and connected, with real analytic boundary, a positive answer for global (that is, when $\Gamma=\partial M$ ) and partial data has been given in $[33,34,36]$. Similarly, the global anisotropic Calderón problem has been answered positively for compact connected Einstein manifolds with boundary in [16].

If the background metrics are not assumed to be analytic, the general anisotropic Calderón problem in dimension $n \geqslant 3$ is still a difficult open problem, whether one is dealing with the case of full or partial data. Nevertheless, some important results have recently appeared in $[10,11,29]$ for special classes of smooth compact connected admissible Riemannian manifolds with boundary. By definition, admissible manifolds $(M, g)$ are conformally transversally anisotropic,

$$
M \subset \subset \mathbb{R} \times M_{0}, \quad g=c\left(e \oplus g_{0}\right),
$$

where $\left(M_{0}, g_{0}\right)$ is an $(n-1)$-dimensional smooth compact connected Riemannian manifold with boundary, $e$ is the Euclidean metric on the real line and $c$ is a smooth strictly positive function in the cylinder $\mathbb{R} \times M_{0}$. It has been shown in $[10,11]$ that for admissible manifolds and under some geometric assumptions on the transverse manifolds $M_{0}$ (see for instance [11] for a precise statement), the conformal factor $c$ is uniquely determined from the knowledge of the DN map. These results have been further extended to the case of partial data in [29]. We also refer to $[17,23,25]$ for additional results in the case of local data and to surveys $[18,30]$ for further references.

Let us also mention several papers dealing with the Calderón problem for more singular metrics or conductivities in dimension $n \geqslant 3$. Haberman and Tataru [20] showed uniqueness in the global Calderón problem for uniformly elliptic isotropic conductivities that are Lipschitz and close to the identity. The latter condition was relaxed by Caro and Rogers in [5]. In dimensions three and four, these results were slightly improved by Haberman in [19] to the case of conductivities that belong to $W^{1, n}$. Related to the partial Calderón problem, Krupchyk and Uhlmann in [32] proved that an isotropic conductivity with—roughly speaking- $\frac{3}{2}$ derivatives in the $L^{2}$ sense is uniquely determined by a DN map measured on a possibly very small subset of the boundary.

Finally, we conclude this introduction mentioning the series of papers on invisibility by Greenleaf, Kurylev, Lassas and Uhlmann (see [15] for the original paper, [3] for an extension of this work in dimension two and [14, 45] for thorough surveys in this field). In these works, some counterexamples to uniqueness to the global Calderón problem were described. These counterexamples are obtained for a class of metrics that are highly singular at a given closed hypersurface 
lying within the manifold in the sense that the metric degenerates or blows up at this hypersurface. This is a situation in sharp contrast to the one obtained in the present work in which the metrics remain positive definite and Hölder continuous everywhere in the manifold (and even smooth in the interior). Coming back to invisibility, we also refer to our recent paper [9], where similar nonuniqueness results were obtained for singular warped product metrics on the same class of manifolds $M$ as the ones used in this paper.

\section{Counterexamples to uniqueness}

2.1. Miller's counterexample to the unique continuation principle. In this section, we recall the remarkable counterexamples obtained by Miller [39] to the unique continuation principle, counterexamples that were slightly improved later by Mandache [38]. We say that a $\operatorname{PDE} P(x, D) u=0$ on a domain $\Omega$ possesses the unique continuation property if the equality $u=0$ in some ball within $\Omega$ implies the equality $u=0$ on $\Omega$.

In dimension $n \geqslant 3$, the unique continuation property holds for a uniformly elliptic operator on a domain $\Omega$ if the coefficients of the principal part of this operator are locally Lipschitz continuous (see for instance [21, 22, 42]). If the coefficients are only Hölder continuous, then there exist examples of nonunique continuation. The first one was given in 1963 by Pliś [40]. He considered a uniformly elliptic equation on a domain of $\mathbb{R}^{3}$ having the form

$$
\sum_{i, j=1}^{3} a_{i j}(x) \partial_{i j}^{2} u+\sum_{i=1}^{3} b_{i}(x) \partial_{i} u+c(x) u=0,
$$

where the coefficients of this equation are Hölder continuous with order less than 1. The coefficient of the zero-order term $c(x)$ has no constant sign and might explain the existence of this counterexample. In 1972, a sharper counterexample (without the zero-order term) was found by Miller [39]. He constructed a smooth solution $u(t, x, y)$ of a uniformly elliptic equation in divergence form:

$$
\operatorname{div}(\mathcal{A} \nabla u)=0
$$

where the $(3 \times 3)$ symmetric matrix $\mathcal{A}$ is given by

$$
\mathcal{A}=\left(\begin{array}{ccc}
1 & 0 & 0 \\
0 & 1+a_{1}(t, x, y)+A_{1}(t) & a_{2}(t, x, y) \\
0 & a_{2}(t, x, y) & 1+a_{3}(t, x, y)+A_{3}(t)
\end{array}\right) .
$$

This matrix $\mathcal{A}$ has its eigenvalues in $\left[\alpha, \alpha^{-1}\right]$ with elliptic constant $\left.\alpha \in\right] 0,1[$. More precisely, Miller proved the following result. 
THEOREM 2 (Miller [39]). There exists an example of nonunique continuation on the half-space $E=\left[0,+\infty\left[\times \mathbb{R}^{2}\right.\right.$ for a uniformly elliptic equation

$$
\begin{aligned}
& \partial_{t}^{2} u+\partial_{x}\left(\left(1+a_{1}+A_{1}\right) \partial_{x} u\right)+\partial_{x}\left(a_{2} \partial_{y} u\right)+\partial_{y}\left(a_{2} \partial_{x} u\right)+\partial_{y}\left(\left(1+a_{3}+A_{3}\right) \partial_{y} u\right) \\
& \quad=0 \text { in } E .
\end{aligned}
$$

(1) The classical solution $u(t, x, y)$ is $C^{\infty}$ on $E$, identically zero for $t \geqslant T>0$, but not identically zero in any open subset of $\left[0, T\left[\times \mathbb{R}^{2}\right.\right.$.

(2) The coefficients $a_{1}(t, x, y), a_{2}(t, x, y), a_{3}(t, x, y)$ are $C^{\infty}$ on $E$ and are identically zero for $t \geqslant T$.

(3) The coefficients $A_{1}(t), A_{3}(t)$ are Hölder continuous of order $\frac{1}{6}$ on $[0, \infty[$, $C^{\infty}$ on $[0, T[$, and identically zero for $t \geqslant T$.

(4) All functions $u, a_{1}, a_{2}, a_{3}$ are periodic in $x$ and $y$ with period $2 \pi$.

(5) Although the coefficient matrix $\mathcal{A}$ is only Hölder continuous at $t=T$, $u$ is a classical (as well as weak) solution of (2.34) on E.

We emphasize that this theorem can be improved as follows. Modifying slightly Miller's initial proof, the coefficients $A_{1}(t), A_{3}(t)$ can be actually constructed in such a way that they are Hölder continuous functions of fixed order $\rho<1$ (see [39], Remarks p. 115) as in Mandache's paper [38]. But, we prefer to use Miller's work rather than Mandache's paper [38] since it is not clear for us that the coefficients of the elliptic operator constructed by Mandache are smooth on $[0, T$ [. It is also important to say again that this function $u$ is a classical solution of elliptic equation (2.34) since we do not differentiate the Hölder functions $A_{1}(t)$ and $A_{3}(t)$ with respect to $t$ in elliptic equation (2.34).

Moreover, since the solution $u(t, x, y)$ is periodic in $(x, y)$ with period $2 \pi$, as was observed by Giannotti in [12], Miller's solution can be considered as a solution to an elliptic equation on the toroidal ring $M=[0, T] \times T^{2}$, where $T^{2}$ is the usual two-dimensional torus.

Note that, in the following section, and in order to simplify the notation, we assume (without loss of generality) that $T=1$.

2.2. Construction of counterexamples on a toroidal ring of $\mathbb{R}^{\mathbf{3}}$. In this section, we consider a Riemannian manifold $(M, g)$, which has the topology of a cylinder $M=[0,1] \times T^{2}$. We denote by $(t, x, y)$ a global coordinate system on $M$. The manifold $M$ can be interpreted as a toroidal ring (see [12], Remark 2.5). We equip this manifold with the Riemannian metric

$$
g=D d t^{2}+\left(1+a_{3}+A_{3}\right) d x^{2}-2 a_{2} d x d y+\left(1+a_{1}+A_{1}\right) d y^{2},
$$


where the coefficients $a_{1}, a_{2}, a_{3}, A_{1}, A_{3}$ are given by Theorem 2, and

$$
D=\operatorname{det} \mathcal{A}=\left(1+A_{1}(t)+a_{1}(t, x, y)\right)\left(1+A_{3}(t)+a_{3}(t, x, y)\right)-a_{2}^{2}(t, x, y) .
$$

Observe that we have $\sqrt{|g|}\left(g^{-1}\right)=\mathcal{A}$ and that this metric is well defined on $M$ since all the coefficients are periodic in the variables $x, y$ with period $2 \pi$. We recall that the boundary $\partial M$ of $M$ is disconnected and consists in the disjoint union of two copies of $T^{2}$, precisely,

$$
\partial M=\Gamma_{0} \cup \Gamma_{1}, \quad \Gamma_{0}=\{0\} \times T^{2}, \quad \Gamma_{1}=\{1\} \times T^{2} .
$$

We emphasize that our metric $g$ is smooth everywhere inside the manifold (precisely on $\bar{M} \backslash \Gamma_{1}$ ) and Hölder continuous of order $\rho<1$ on the end $\Gamma_{1}$. Thanks to Theorem 2 and as was observed before, Miller's solution is a classical harmonic function for the Laplace-Beltrami operator $\Delta_{g}$, that is, it satisfies the Laplace equation in the classical sense

$$
\Delta_{g} u=0 \quad \text { in } M .
$$

Moreover, since the solution $u(t, x, y)$ is smooth on $E$ and is vanishing for $t \geqslant 1$, all the derivatives of $u$ are also equal to zero at $t=1$. In particular, one has

$$
u_{\mid \Gamma_{1}}=0, \quad \partial_{\nu} u_{\mid \Gamma_{1}}=0
$$

where $\partial_{v}$ is the normal derivative on $\Gamma_{1}$ with respect to the unit outer normal vector $v$ on $\partial M$.

Now, let us define our infinite family of conformal factors $c_{\epsilon}(t, x, y)$. We set

$$
c_{\epsilon}(t, x, y)=1+\epsilon u(t, x, y),
$$

where $\epsilon>0$ ranges over an interval $\left(0, \epsilon_{0}\right)$ chosen sufficiently small to ensure that $c_{\epsilon}(t, x, y) \geqslant \frac{1}{2}$ on the compact manifold $M$ for all $\epsilon \in\left(0, \epsilon_{0}\right)$.

Proof of Theorem 1 in dimension three. The conformal factors $c_{\epsilon}$ are smooth on $M$. Moreover, thanks to Theorem 2, they are not identically 1 on $M$. Using (2.37) and (2.38), these conformal factors $c_{\epsilon}$ satisfy obviously and in the classical sense:

$$
\Delta_{g} c_{\epsilon}=0 \quad \text { in } M, \quad c_{\epsilon \mid \Gamma_{1}}=1, \quad \partial_{\nu} c_{\epsilon \mid \Gamma_{1}}=0 .
$$

Then, thanks to Green's formula and using the fact that we have $c_{\epsilon}^{n-2}=c_{\epsilon}$ when $n=3$, these conformal factors $c_{\epsilon}$ satisfy the assumptions of Proposition 1.2, and thus one obtains immediately

$$
\Lambda_{c_{\epsilon}^{4} g, \Gamma_{1}}=\Lambda_{g, \Gamma_{1}} .
$$


Now assume that, for all $0<\epsilon_{1} \leqslant \epsilon_{0}$, there exist an $\epsilon \in\left(0, \epsilon_{1}\right)$ and a diffeomorphism (depending on $\epsilon$ ) $\phi: M \longrightarrow M$ such that $\phi_{\mid \Gamma_{1}}=$ Id and $\phi^{*} g=c_{\epsilon}^{4} g$. In particular, since $\phi$ is a diffeomorphism, we see that $\operatorname{Vol}_{g}(M)=$ $\operatorname{Vol}_{\phi^{*} g}(M)=\operatorname{Vol}_{c_{\epsilon}^{4} g}(M)$. Hence we must have

$$
\int_{M}\left[(1+\epsilon u)^{6}-1\right] \sqrt{|g|} d x=0 .
$$

The term of order 2 of this polynomial in the variable $\epsilon$ must be equal to 0 , that is,

$$
\int_{M} u^{2} \sqrt{|g|} d x=0
$$

which is not possible since $u$ is not identically equal to zero. Thus, we deduce that there exists $0<\epsilon_{1} \leqslant \epsilon_{0}$ such that the metrics $g$ and $c_{\epsilon}^{4} g$ are not isometric for all $\epsilon \in\left(0, \epsilon_{1}\right)$, and we see that we have found counterexamples to uniqueness for the partial anisotropic Calderón problem in dimension three.

2.3. Generalization in the $\boldsymbol{n}$-dimensional case. The above construction can be generalized in a straightforward way to the $n$-dimensional case with $n \geqslant 3$. Indeed, let us consider the manifold $M=[0,1] \times T^{n-1}$, where $T^{n-1}$ stands for the $(n-1)$-dimensional torus. We denote by $\left(t, x_{1}, x_{2}, \ldots, x_{n-1}\right)$ a global coordinate system on $M$. As in the previous section, we introduce the coefficient

$$
D\left(t, x_{1}, x_{2}\right)=\left(1+A_{1}(t)+a_{1}\left(t, x_{1}, x_{2}\right)\right)\left(1+A_{3}(t)+a_{3}\left(t, x_{1}, x_{2}\right)\right)-a_{2}^{2}\left(t, x_{1}, x_{2}\right),
$$

where the coefficients $a_{1}, a_{2}, a_{3}, A_{1}, A_{3}$ are still given by Theorem 2 with the identifications $x_{1}=x$ and $x_{2}=y$. Now, we equip this manifold $M$ with the Riemannian metric

$$
\begin{aligned}
g= & D^{\frac{1}{n-2}}\left(d t^{2}+D^{-1}\left(\left(1+A_{3}+a_{3}\right) d x_{1}^{2}-2 a_{2} d x_{1} d x_{2}\right.\right. \\
& \left.\left.+\left(1+A_{1}+a_{1}\right) d x_{2}^{2}\right)+\sum_{i=3}^{n-1} d x_{i}^{2}\right) .
\end{aligned}
$$

As before, the metric $g$ is smooth inside the manifold $M$ and only Hölder continuous at the end $\Gamma_{1}=\{1\} \times T^{n-1}$. Then, we define the conformal factors (which will not depend on the variables $x_{i}$ for $i \geqslant 3$ ) as

$$
c_{\epsilon}\left(t, x_{1}, \ldots, x_{n-1}\right)=\left(1+\epsilon u\left(t, x_{1}, x_{2}\right)\right)^{\frac{1}{n-2}},
$$


where $u\left(t, x_{1}, x_{2}\right)$ is Miller's solution given in Theorem 2 and $\epsilon>0$ is small enough to ensure that $c_{\epsilon}>0$ on $M$. Using the same arguments as in the previous section, one has

$$
\Lambda_{c_{\epsilon}^{4} g, \Gamma_{1}}=\Lambda_{g, \Gamma_{1}},
$$

which implies that there is nonuniqueness for the partial anisotropic Calderón problem in dimension $n \geqslant 3$.

REMARK 2.1. In the previous nonuniqueness results for the anisotropic Calderón problem with partial data, we considered smooth compact connected cylindrical manifolds equipped with a metric whose coefficients are only Hölder continuous, and having two ends. If we remove the assumption of smoothness for the manifold, then we can allow a connected boundary for $M$ and obtain probably counterexamples to uniqueness in the partial Calderón problem.

More precisely, let us consider the product manifold $M=[0,1] \times \bar{\Omega}$, where $\Omega$ is any connected bounded open set of $\mathbb{R}^{n-1}$ with a smooth boundary. Note that the boundary of $M$ is now connected and given by

$$
\partial M=(\{0\} \times \bar{\Omega}) \cup(\{1\} \times \bar{\Omega}) \cup((0,1) \times \partial \Omega) .
$$

Clearly, we lose the smoothness of the manifold since $M$ has corners. Nevertheless, if the Dirichlet and the Neumann data are measured on $\Gamma=\{1\} \times \bar{\Omega}$, one can probably use the previous constructions to get counterexamples in this new setting.

\section{Acknowledgements}

Thierry Daudé is supported by the JCJC French National Research Projects Horizons, No. ANR- ANR-16-CE40-0012-01, Niky Kamran is supported by NSERC grant RGPIN 105490-2018 and François Nicoleau is supported by the French National Research Project GDR Dynqua. We would like to thank the referee for a careful reading of our manuscript and for providing valuable suggestions.

Conflict of Interest: None.

\section{References}

[1] G. Alessandrini, M. V. De Hoop and R. Gaburro, 'Uniqueness for the electrostatic inverse boundary value problem with piecewise constant anisotropic conductivities', Inverse Problems 33(12) (2017), 125013. 
[2] K. Astala, M. Lassas and L. Paivarinta, 'Calderón's inverse problem for anisotropic conductivities in the plane', Comm. Partial Differential Equations 30 (2005), 207-224.

[3] K. Astala, M. Lassas and L. Päivärinta, 'The borderlines of invisibility and visibility in Calderón's inverse problem', Anal. PDE 9(1) (2016), 43-98.

[4] K. Astala and L. Paivarinta, 'Calderón's inverse conductivity problem in the plane', Ann. of Math. (2) 163 (2006), 265-299.

[5] P. Caro and K. M. Rogers, 'Global uniqueness for the Calderón problem with Lipschitz conductivities', Forum Math. Pi 4 (2016), e2, 28 pp.

[6] T. Daudé, N. Kamran and F. Nicoleau, 'Non uniqueness results in the anisotropic Calderón problem with Dirichlet and Neumann data measured on disjoint sets, (2015)', Ann. Inst. Fourier (Grenoble) 69(1) (2019), 119-170.

[7] T. Daudé, N. Kamran and F. Nicoleau, 'On the hidden mechanism behind non-uniqueness for the anisotropic Calderón problem with data on disjoint sets, (2017)', Ann. Henri Poincaré 20 (3) (2019), 859-887.

[8] T. Daudé, N. Kamran and F. Nicoleau, 'A survey of non-uniqueness results for the anisotropic Calderón problem with disjoint data', in Harvard CMSA Series in Mathematics, Volume 2: Nonlinear Analysis in Geometry and Applied Mathematics (eds. T. Collins and S.-T. Yau) (International Press, Boston, 2018).

[9] T. Daudé, N. Kamran and F. Nicoleau, 'The anisotropic Calderón problem for singular metric of warped product type: the borderline between uniqueness and invisibility', J. Spectr. Theory, to appear, arXiv:1805.05627.

[10] D. Dos Santos Ferreira, C. E. Kenig, M. Salo and G. Uhlmann, 'Limiting Carleman weights and anisotropic inverse problems', Invent. Math. 178(1) (2009), 119-171.

[11] D. Dos Santos Ferreira, Y. Kurylev, M. Lassas and M. Salo, 'The Calderón problem in transversally anisotropic geometries', J. Eur. Math. Soc. (JEMS) 18(11) (2016), 2579-2626.

[12] C. Giannotti, 'A compactly supported solution to a three-dimensional uniformly elliptic equation without zero-order term', J. Differential Equations 201 (2004), 234-249.

[13] D. Gilbarg and N. N. Trudinger, Elliptic Partial Differential Equations of Second Order, Classics in Mathematics (Springer, Berlin, 2001), xiv+517 pp. Reprint of the 1998 edition.

[14] A. Greenleaf, Y. Kurylev, M. Lassas and G. Uhlmann, 'Invisibility and inverse problems', Bull. Amer. Math. Soc. (N.S.) 46(1) (2009), 55-97.

[15] A. Greenleaf, M. Lassas and G. Uhlmann, 'On non-uniqueness for Calderón's inverse problem', Math. Res. Lett. 10(5) (2003), 685-693.

[16] C. Guillarmou and A. Sà Barreto, 'Inverse problems for Einstein manifolds', Inverse Probl. Imaging 3 (2009), 1-15.

[17] C. Guillarmou and L. Tzou, 'Calderón inverse problem with partial data on Riemann surfaces', Duke Math. J. 158(1) (2011), 83-120.

[18] C. Guillarmou and L. Tzou, 'The Calderón inverse problem in two dimensions', in Inverse Problems and Applications: Inside Out. II, Mathematical Sciences Research Institute Publications, series 60 (Cambridge University Press, Cambridge, 2013), 119-166.

[19] B. Haberman, 'Uniqueness in Calderón's problem for conductivities with unbounded gradient', Comm. Math. Phys. 340(2) (2015), 639-659.

[20] B. Haberman and D. Tataru, 'Uniqueness in Calderón's problem with Lipschitz conductivities', Duke Math. J. 162(3) (2013), 496-516.

[21] L. Hörmander, The Analysis of Linear Partial Differential Operators, III, Grundlehren der matematischen Wissenschaften, 256 (Springer, Berlin, Heidelberg, 1985).

[22] L. Hörmander, 'Uniqueness theorems for second order elliptic differential equations', Comm. Partial Differential Equations 8(1) (1983), 21-64. 
[23] O. Imanuvilov, G. Uhlmann and M. Yamamoto, 'The Calderón problem with partial data in two dimensions', J. Amer. Math. Soc. 23 (2010), 655-691.

[24] O. Y. Imanuvilov, G. Uhlmann and M. Yamamoto, 'Inverse boundary value problem by measuring Dirichlet data and Neumann data on disjoint sets', Inverse Problems 27(8) (2011), 085007, 26p.

[25] V. Isakov, 'On uniqueness in the inverse conductivity problem with local data', Inverse Probl. Imaging 1 (2007), 95-105.

[26] H. Kang and K. Yun, 'Boundary determination of conductivities and Riemannian metrics via local Dirichlet-to-Neumann operators', SIAM J. Math. Anal. 34(3) (2003), 719-735.

[27] A. Katchalov, Y. Kurylev and M. Lassas, Inverse boundary spectral problems, Monographs and Surveys in Pure and Applied Mathematics, 123 (Chapman Hall/CRC, 2001).

[28] A. Katchalov, Y. Kurylev, M. Lassas and N. Mandache, 'Equivalence of time-domain inverse problems and boundary spectral problem', Inverse Problems 20 (2004), 419-436.

[29] C. Kenig and M. Salo, 'The Calderón problem with partial data on manifolds and applications', Anal. PDE 6(8) (2013), 2003-2048.

[30] C. Kenig and M. Salo, 'Recent progress in the Calderón problem with partial data', Contemp. Math. 615 (2014), 193-222.

[31] C. Kenig, J. Sjöstrand and G. Uhlmann, 'The Calderón problem with partial data', Ann. of Math. (2) 165 (2007), 567-591.

[32] K. Krupchyk and G. Uhlmann, 'The Calderón problem with partial data for conductivities with 3/2 derivatives', Comm. Math. Phys. 348(1) (2016), 185-219.

[33] M. Lassas, G. Taylor and G. Uhlmann, 'The Dirichlet-to-Neumann map for complete Riemannian manifolds with boundary', Comm. Anal. Geom. 11 (2003), 207-221.

[34] M. Lassas and G. Uhlmann, 'On determining a Riemannian manifold from the Dirichlet-toNeumann map', Ann. Sci. Éc. Norm. Supér. (4) 34 (2001), 771-787.

[35] J. M. Lee, Introduction to Smooth Manifolds, 2nd edn, Graduate Texts in Mathematics (Springer, New York, 2000).

[36] J. M. Lee and G. Uhlmann, 'Determining anisotropic real-analytic conductivities by boundary measurements', Comm. Pure Appl. Math. 42(8) (1989), 1097-1112.

[37] W. R. B. Lionheart, 'Conformal uniqueness results in anisotropic electrical impedance imaging', Inverse Problems 13 (1997), 125-134.

[38] N. Mandache, 'On a counterexample concerning unique continuation for elliptic equations in divergence form', Mat. Fiz. Anal. Geom. 3(3/4) (1996), 308-331.

[39] K. Miller, 'Non-unique continuation for uniformly parabolic and elliptic equations in selfadjoint divergence form with Hölder continuous coefficients', Arch. Ration. Mech. Anal. 54(2) (1974), 105-117.

[40] A. Pliś, 'On non-uniqueness in Cauchy problem for an elliptic second order differential equation', Bull. Acad. Pol. Sci. S. Mat. XI(3) (1963), 95-100.

[41] M. Salo, 'The Calderón problem on Riemannian manifolds', in Inverse Problems and Applications: Inside Out. II, Mathematical Sciences Research Institute Publications, 60 (Cambridge University Press, Cambridge, 2013), 167-247.

[42] D. Tataru, 'Unique continuation for PDEs', IMA Vol. Math. Appl. 137 (2003), 239-255.

[43] M. Taylor, Partial Differential Equations. I. Basic Theory, Applied Mathematical Sciences, 115 (Springer, New York, 2011).

[44] G. Uhlmann, 'Electrical impedance tomography and Calderón's problem', Inverse Problems 25123011 (2009), 39p.

[45] G. Uhlmann, 'Inverse problems: seeing the unseen', Bull. Math. Sci. 4(2) (2014), 209-279. 